IP Periodica Polytechnica

Transportation Engineering

46(1), pp. 29-35, 2018

https://doi.org/10.3311/PPtr. 10753

Creative Commons Attribution (i)

RESEARCH ARTICLE

\section{Development of a Test Track for Driverless Cars: Vehicle Design, Track Configuration, and Liability Considerations}

\author{
Zsolt Szalay ${ }^{1}$, Tamás Tettamanti ${ }^{2 *}$, Domokos Esztergár-Kiss ${ }^{3}$, \\ István Varga ${ }^{2}$, Cesare Bartolini ${ }^{4}$
}

Received 18 January 2017; accepted 18 March 2017

\begin{abstract}
The Research Center for Autonomous Road Vehicles (RECAR) was founded in 2015 upon the initiative of the Faculty of Transportation Engineering and Vehicle Engineering of Budapest University of Technology and Economics. The research center is supported by industrial partners and other academic partners targeting research and educational purposes. In complement to this project, the construction of a new automotive test track is also under development especially for autonomous road vehicle testing serving as automotive proving ground in Zalaegerszeg, Hungary. Accordingly, an intensive research has been started in RECAR center in the field of autonomous vehicle technology. The paper's goal is to share the main practical and methodological experiences with the scientific audience as well as the industrial sector. Based on the initial research actions we intend to enlighten the upcoming research challenges of driverless vehicles and automated intelligent transport system. Basically, three main topics are concerned. Firstly, the main issues concerning autonomous vehicle research are summarized. Secondly, the requirements for autonomous test track design are concluded. Thirdly, the legal questions that emerge with the appearance of driverless vehicles are investigated, especially concerning liability.
\end{abstract}

\section{Keywords}

autonomous vehicle, vehicle design, automated intelligent transport system, automotive test track, liability

\footnotetext{
${ }^{1}$ Department of Automotive Technologies, Faculty of Transportation Engineering and Vehicle Engineering, Budapest University of Technology and Economics, Stoczek J. u. 6., 1111 Budapest, Hungary

${ }^{2}$ Department of Control for Transportation and Vehicle Systems, Faculty of Transportation Engineering and Vehicle Engineering, Budapest University of Technology and Economics, Stoczek J. u. 2., 1111 Budapest, Hungary

${ }^{3}$ Department Transport Technology and Economics, Faculty of Transportation Engineering and Vehicle Engineering, Budapest University of Technology and Economics, Stoczek J. u. 2., 1111 Budapest, Hungary

${ }^{4}$ Interdisciplinary Centre for Security, Reliability and Trust, University of Luxembourg, rue Alphonse Weicker, L-2721 Luxembourg

*Corresponding author, e-mail: tettamanti@mail.bme.hu
}

\section{Introduction}

Connected and automated vehicle technology will basically transform our life in the near future (SAE International, 2014; Gáspár et al., 2014; Törő et al., 2016). The general impact of autonomous transport has been examined in several recent papers. The major social impacts have been summarized as the gains due to safer roads, travel time reduction, more personalized services, improvement of energy efficiency and parking benefits (Fagnant and Kockelman, 2015). The most important inducements are the safety-related issues. An overwhelming majority of the accidents (app. 95\%) are caused by human imperfection. It is estimated that self-driving technology could eliminate $90 \%$ of road traffic accidents (Waldrop, 2015). Moreover, Schoettle and Sivak (2015) found that self-driving vehicles were not faulty in any crashes they were involved in.

Market researches outline different scenarios regarding the penetration of autonomous cars. Bansal and Kockelman (2017) concluded that the promotion of the technology and rapid reductions in technology costs would be the main elements necessary for a significant diffusion of driverless cars. Milakis et al. (2017) introduced a scenario matrix concerning the development of automated vehicles, assuming combinations of high or low technological development, and restrictive or supportive policies for automated vehicle. Even the worst case scenario given in (Milakis et al., 2017) predicts the spread of fully autonomous in 2045 at the latest. Nevertheless, it is straightforward that in the next decades a significant part of road vehicles will be equipped by autonomous functionalities (Fagnant and Kockelman, 2015).

Accordingly, the research and development of autonomous vehicle technology and related topics are important and require extensive work in the future. Therefore, the Research Center for Autonomous Road Vehicles (RECAR) was founded upon the initiative of the Faculty of Transportation Engineering and Vehicle Engineering of Budapest University of Technology and Economics, supported by industrial and other academic partners. The aim of this paper is to provide the first experiences and basic findings of the research center, and the related automotive test track design. 
The paper is structured as follows. In the second chapter, the main and goals of the new autonomous research center are described. As the main focus of the new establishment is on education and research, the educational structure and also the planned test track is introduced. The main research areas are presented in the third chapter, together with the layers of autonomous vehicle control, which help determine the main research areas. Research testing and validation have great emphasis in the field of autonomous vehicle, therefore the requirements and specifications for test tracks are described in the fourth chapter. Finally, in the fifth chapter, liability concerning driverless vehicles is investigated, which is the prerequisite of commercial applicability of the new functions.

\section{RECAR: research center for autonomous road vehicles}

With the establishment of the REsearch Center for Autonomous Road vehicles (RECAR: http://recar.bme.hu/eng/), the partners created a comprehensive organization in which they combine their competences. With the utilization of synergies, higher-level research results can be achieved. RECAR's aim is to connect and exploit the academic and industrial competences, and also education and research, so that the training of highly-qualified professionals and the strengthening of $\mathrm{R}+\mathrm{D}+\mathrm{I}$ basis can be enabled. As a first step of this roadmap, in January 2016 the leaders of BME (Budapest University of Technology and Economics), ELTE (Eötvös Loránd University) and MTA SZTAKI (Hungarian Academy of Sciences, Institute for Computer Science and Control) signed the collaboration agreement of the RECAR program.

Based on international trends and incentives of national companies in automotive industry, the partners initiated two new master programs (MSc): Autonomous Vehicle Control Engineer hosted by BME and Computer Science for Autonomous Driving hosted by ELTE. The foundations for the master programs have already been laid down and the detailed education plans are on the way, together with the elaboration of course materials. It has to be stressed that the master programs were jointly defined by the academic and industrial partners in order to better follow the real industry needs. The education will be launched in 2018 .

For education and research in the domain of autonomous vehicle technologies, the establishment of laboratories beyond the state-of-the-art is essential. The partners have defined a testing and validation structure of 5 layers (Szalay, 2016), including laboratories that serve both educational and research needs (Fig. 1). The laboratory tests are composed of technology research labs, component analysis labs, system integration labs, and, last but not least, a vehicle-in-the-loop lab. The labs will have unique and high-quality equipment in order to test and validate most of the functionalities for autonomous vehicle developments.

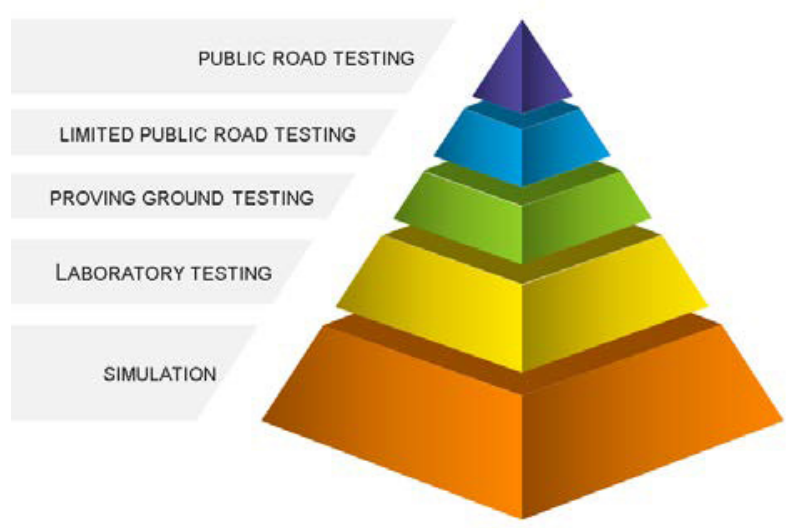

Fig. 1 Autonomous vehicle testing and validation layers (Szalay, 2016)

Directly linked to the research center, a new test track will be realized in Hungary (close to the city of Zalaegerszeg) through an investment of 130 million Euros by 2019. The planned proving ground (Fig. 2) is especially dedicated to testing connected and autonomous vehicle functionalities even in urban environment (with general roadside objects, city traffic elements, building facades and traffic infrastructure). The 250 ha area will incorporate the following test features:

- standard vehicle dynamics testing and validation,

- fully integrated autonomous vehicle testing and validation,

- environment preparation (obstacles, traffic signs, traffic control, other vehicles, vulnerable road users),

- complex driving and traffic situations,

- smart city features,

- testing and validation, from prototype testing to mass production.

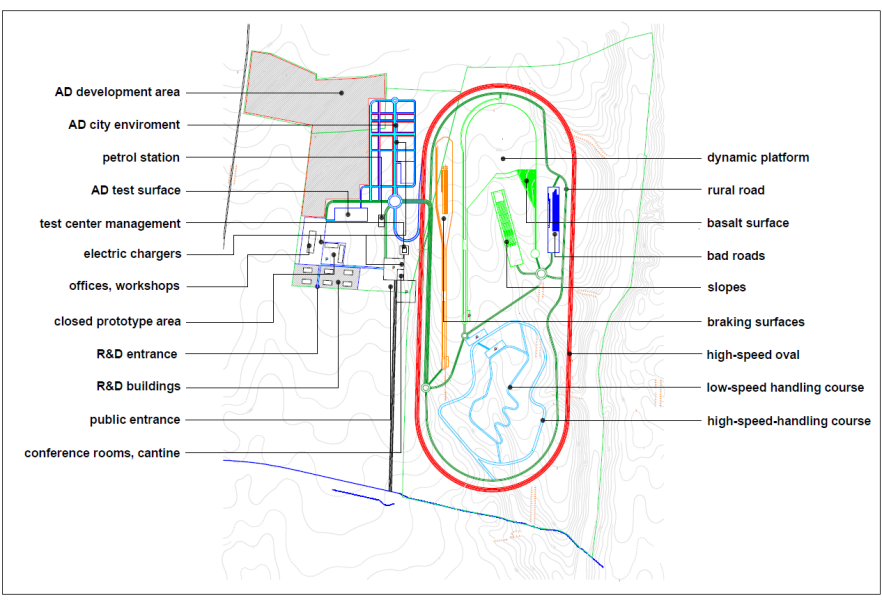

Fig. 2 Automotive proving ground dedicated to autonomous vehicle testing and validation at Zalaegerszeg (AD in figure means Automated Driving)

The proving ground should be suitable for a wide range of vehicle and traffic test scenarios for conventional, connected and automated vehicles. Test capabilities will cover not only prototype testing, but also type-approval procedures and educational purposes. One of the possible future use cases for testing will be the autonomous driving (i.e. SAE level 5 
(SAE International, 2014)) from suburban home to city office together with autonomous parking (rural road, highway traffic, sub-urban area, city environment with continuous transition) (Szalay, 2016).

\section{Main scope of autonomous vehicle research}

The research and development in the domain of highly-automated or autonomous road vehicle requires a well-defined and separated structure which consists of environment perception, potential trajectory analysis, decision making and decision execution. All these need to happen in real time and in a fault-tolerant, or at least fail-safe way. Researchers and vehicle manufacturers may have different implementations of the control structure (see the HAVEit structure as an example in Fig. 3), but all of them have to ensure the following four layers for integrated vehicle control as a minimum requirement:

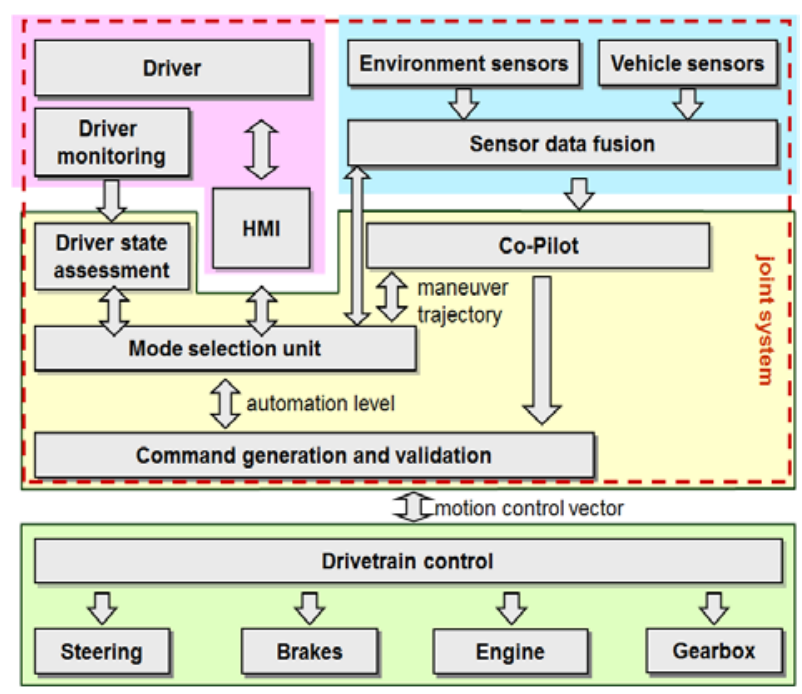

Fig. 3 Example of an integrated vehicle control structure (source: Hoeger et al., 2011)

1. The driver interface layer is not only responsible for interacting with the driver about the actual status of the autonomously-running vehicle, but it also has to continuously evaluate the driver's status and his/her capability to take over control in case a fallback performance is required.

2. The environment perception layer is responsible for providing comprehensive information about the traffic situation, including the objects surrounding the vehicle. There are different types of sensors installed all around the vehicle, such as sonars, radars, lidars, video cameras and laser scanners. They are combined with an e-Horizon based location data (GPS/Glonass/BeiDou, etc.) and high-definition mapping information. Since different sensors are working based on different phenomena, the confidence level of the output data strongly depends on the environmental (e.g., weather) conditions they operate under. That is why sensor fusion algorithms are of key importance in generating reliable situation awareness and environmental information.

3. Depending on the automation level, the trajectory planning layer calculates possible vehicle trajectories with priority ranks of performance and safety. It involves the calculation of longitudinal and lateral trajectory options, different route possibilities with respect to the surrounding environment, the ranking and prioritization of the different route options based on minimizing the risk of a collision, and ends up in the selection of the optimum trajectory.

4. The trajectory execution layer receives the selected trajectory as an input from the planning layer. After the trajectory segmentation and the motion vector generation (containing longitudinal and lateral control commands), the execution of the motion vector is performed by distributing it among the intelligent actuators of the vehicle drivetrain.

By considering the four layers given above, and exploiting the special functionalities of the RECAR laboratories, several new research projects are planned to initiate in the near future. As a preliminary step, the main research fields of autonomous vehicle technology had to be identified. The research topics are motivated by up-to-date international trends and initiatives of industrial partners. The structure of the research topics also reflects the recent EU actions by means of the Gear 2030 body activities towards formulating a harmonized and competitive European vision for the "Connected Car and Automated Driving".

Finally, 7 main research groups have been identified (tabulated into Table 1). According to Table 1, future works will be realized in the form of continuous industrial projects and research collaborations.

\section{Requirements for autonomous test track design}

Several test tracks are available in the automotive industry serving as proving grounds for traditional road vehicles. However, the test track requirements are only partly identical for autonomous vehicle technologies. To the best of our knowledge, only one publicly available proving ground exists in the world, which has been specifically designed for autonomous vehicle needs. That proving ground is the Mobility Transformation Center of University of Michigan, USA, or Mcity for short. Therefore, the new Hungarian test track (mentioned in chapter 2) will represent a unique facility in Europe by providing OEM ${ }^{1}$-independent capability for the testing and validation of connected and automated vehicles and systems. Accordingly, the proper requirement definition for the test track is extremely important. This task, however, is not straightforward at all. No basic specification has been published so

1 Original Equipment Manufacturer. 
Table 1 The identified research groups with the main features

\begin{tabular}{|c|c|}
\hline Research group title & Main features of the research group \\
\hline $\begin{array}{l}\text { 1. Development of autonomous } \\
\text { vehicle demonstration platform }\end{array}$ & 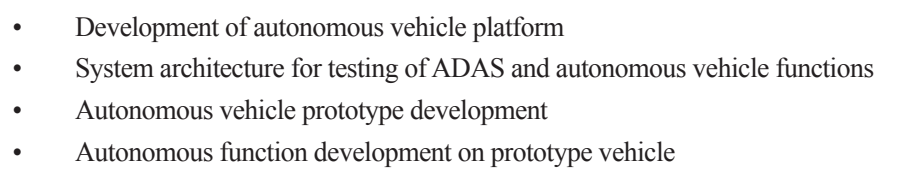 \\
\hline 2. Control of autonomous vehicles & $\begin{array}{l}\text { - } \quad \text { Cooperative navigation based on environmental and vehicle sensors } \\
\text { - } \quad \text { Cooperative and distributed vehicle control strategies } \\
\text { - } \quad \text { Optimal trajectory planning considering static and dynamic obstacles } \\
\text { - } \quad \text { Model-driven design tools and techniques for autonomous vehicles } \\
\text { - Decision-making and action modeling in different traffic situations }\end{array}$ \\
\hline $\begin{array}{l}\text { 3. Communication systems within } \\
\text { and among vehicles }\end{array}$ & 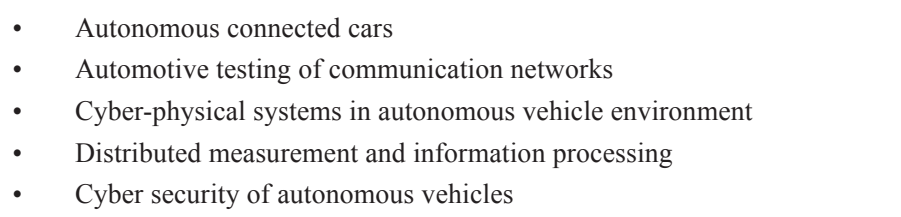 \\
\hline $\begin{array}{l}\text { 4. Environment sensing of } \\
\text { autonomous vehicles }\end{array}$ & $\begin{array}{l}\text { - } \quad \text { Environment sensing based on multisensor fusion } \\
\text { - } \quad \text { Optimal sensor architecture for autonomous vehicles } \\
\text { - } \quad \text { Signal processing of environment sensing and visualization } \\
\text { - } \quad \text { High-definition mapping for autonomous vehicles }\end{array}$ \\
\hline 5. Intelligent transportation systems & 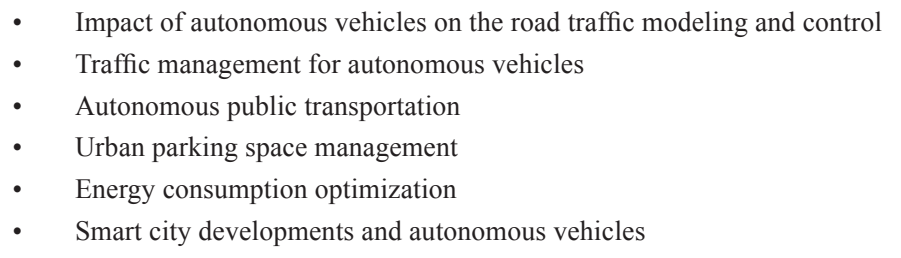 \\
\hline $\begin{array}{l}\text { 6. Interaction of human and } \\
\text { autonomous vehicles }\end{array}$ & $\begin{array}{l}\text { - } \quad \text { Traveler behavior in autonomous public transport } \\
\text { - } \quad \text { Automation possibilities of information management an decision-making } \\
\text { - } \quad \text { Human factors and dynamic characteristics } \\
\text { - } \quad \text { Anomaly detection and prediction using smart devices } \\
\text { - }\end{array}$ \\
\hline $\begin{array}{l}\text { 7. Testing and validation of } \\
\text { autonomous vehicles }\end{array}$ & $\begin{array}{l}\text { - Test and validation on test track and in real world } \\
\text { Process definition for certified testing and validation of autonomous } \\
\text { technologies }\end{array}$ \\
\hline
\end{tabular}

far. One pioneer work has been reported by UK Government (Department of Transport) providing a code of practice for testing driverless vehicles (Hoeger et al., 2011). Nevertheless, this manual can be considered as a reference only in a limited way, as it concerns the testing issues on public roads and not on a test track. Accordingly, in the following, a concise specification is provided for autonomous test field design, together with the special requirements for traffic control system in the test field.

\subsection{General requirements for autonomous test track design}

Several working groups have been set up within the RECAR framework, dedicated to each preparatory work on special tasks. The task of the first working group was the formulation of the technical specification for the proving ground dedicated to connected and automated vehicles. The invited participants for each working group were selected by a thorough stakeholder analysis. The participants in the technical specification working group are classified by their role in the Hungarian automotive sector:

- Industrial companies

- Research centers and universities

- Authorities and governmental bodies

The official meetings started in March 2016 under strict NDA conditions, and specification of the proving ground was finished by the end of November in 2016. The whole process was coordinated by RECAR coordination office.

According to the initial vision, the proving ground will have all the standard endurance testing features like a "conventional" proving ground, but all tracks are designed in such a way that they incorporate all necessary requirements to be able to test 
the vehicles for connected and automated features. In the proving ground design there are workplaces to do simulation work, and also buildings dedicated to special laboratories. The test track is located on the largest area of the facility. Limited public road tests could be performed in a dedicated part of the city of Zalaegerszeg, and this area would incorporate also "Smart City" features. Public road tests could be performed on the highway roads between Budapest and Zalaegerszeg, thanks to an ongoing traffic code modification by the Hungarian government.

The proving ground specification contains detailed requirements for the following special elements:

- Urban tracks for autonomous vehicles: this part has complex city road elements, like signalized intersections, streets, parking places and buildings, and special equipment (e.g., moving obstacles). Smart City features (such as intelligent lightening) are also going to be realized here.

- Rural roads: to test vehicles with lower velocities. The shape of this road is designed to be challenging for autonomous systems.

- General highway road: to test high speed autonomous functions, e.g., lane keeping or lane changing.

- Other alternative grounds to test the vehicles on roads with different qualities, slopes, or to do measurements.

- Dedicated grounds to test Car-to-X (C2X) communication technologies.

In conclusion, all important requirements have been revealed for the Hungarian autonomous test field design through realworld stakeholder involvement (private companies and public institutions).

\subsection{Design requirements concerning traffic control on the test track}

Besides the general design requirements, one significant problem must be investigated. How can an optimal framework for the road traffic control and related issues in the test facility be planned? This is extremely important for driverless vehicle test. On the one hand, traditional traffic light control is needed, as robo-pilots will deal with traffic signals in the transition period (when traditional and autonomous cars will coexist). On the other hand, advanced technologies must be also present, supporting V2I based traffic control. Consequently, traditional traffic lights will expectedly appear as virtual traffic signals inside the vehicles.

Accordingly, requirements and architecture for traffic control system are collected in the following. A potential architecture of road traffic control in the test field is depicted in the figure below. This concept is based on the real-word practice (application of traffic center) with one significant difference. Besides vendor-specific local traffic light controllers and Variable Message Sign (VMS) tables (usually one at each intersection), Programmable Logic Controller (PLC) based control is applied to the system. According to Fig. 4, the main traffic signal components in an autonomous test track would reasonably consist of traffic signal heads, variable message signs, and signals of any kind of hardware controllable via PLC. By means of the PLC technology, signal components could be directly controlled and monitored from a central location (practically from a central control room of the test track).

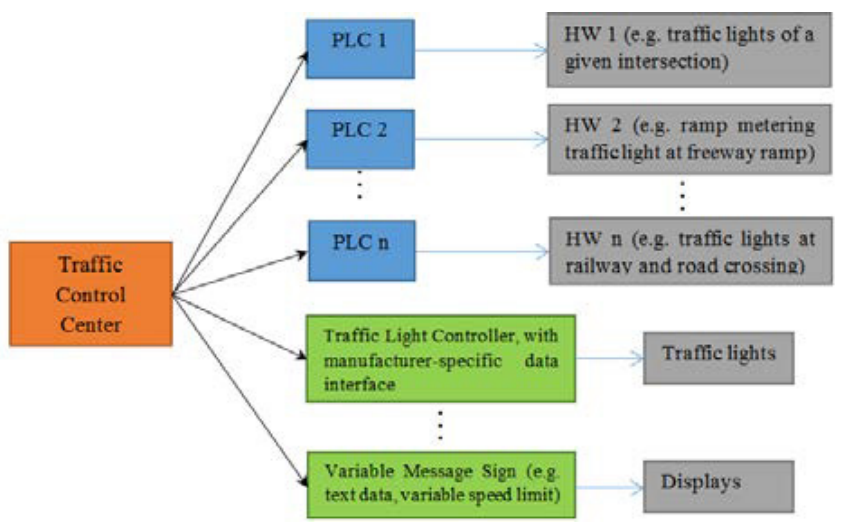

Fig. 4 Road traffic control system architecture in the test track

The use of PLCs for traffic light control is straightforward as PLCs are robust and customizable. They contain electrically-operated switches. PLC relays can directly switch power to any channel (bulb) of traffic signal component. On the other hand, PLCs are able to monitor the power on the channels, thus they can be used for failure detection concerning the signal states of signal heads. This approach enables a maximal flexibility in the test field, i.e., test scenarios at intersections can be set up with an arbitrary number of signal components.

As communication protocol, the UDP (User Datagram Protocol) is suggested between traffic control system and PLC components. If PLCs cannot be applied, a direct control of the traffic lights from the traffic control center is needed with the use of a specific control protocol. Full matrix VMS (capable to display arbitrary text or graphics) cannot be controlled by means of a digital power-switching techniques. These devices can be controlled remotely by using manufacturer-specific protocol. The same is true when a traffic light controller is monitored by the control center, where manufacturer-specific communication protocols must be applied.

The implementation of safety-critical functions of the system (according to the European norm EN 12675:2002) can be ensured at the level of the PLC, of the traffic light controller and of the VMS, and shall satisfy the following requirements:

- recognition of prohibited (conflicting) or false signals;

- switching to fail-safe position state (depending on the error type) in case of internal errors;

- checking the right green light combination: prohibited greens cannot be on at the same time (checking the existence of the intermediate times);

- failure detection of the signal components. 


\section{Liability questions related to driverless cars}

A very relevant topic concerning driverless cars is that of the legal framework. Given that vehicular traffic is one of the mostly regulated domains, with a solid framework of similar regimes across many different countries, the appearance of driverless cars is disruptive.

The main issue that is snapped under the weight of the new technology is that of liability (Marchant and Lindor 2012). Laws have complex regimes governing liability in car accidents. As car driving is universally recognized as a dangerous activity (Harper and Kime, 1934), there is a complex chain of rules $^{2}$ concerning:

- manufacturing requirements and product liability (Stapleton, 1994);

- administrative requirements for car ownership and liability of car owners;

- mandatory insurance for car owners;

- administrative requirements for car driving and liability of the driver (Pascàud, 1908).

In a driverless car, the car occupant cannot be considered a "driver", and therefore the normal rules for driver's licenses and liability are jeopardized. As autonomous cars take over the market and the roads, the rules for vehicular traffic will need to be rewritten.

On the basis of the current landscape, the driver can incur into criminal and civil liability. Given its function as a penalty, criminal liability is bound to the behavior of the agent. Therefore, in the absence of a causal bond between the behavior of the driver and the event, no liability can be placed on the driver. Civil liability, on the other hand, aims at restoring damages suffered in consequence of an accident, so if the damages are independent of the driver's behavior, the remedies might be charged upon someone else, e.g., the manufacturer in case the vehicle was malfunctioning.

As a rule of thumb, the main effect of driverless cars on the existing legal framework is to take the liability off the driver. This has significant consequences from the perspective of administrative rules, civil liability and criminal liability. However, both the market and the legislation are undergoing a long transition phase, during which changes occur gradually.

Eventually, in a context where cars are completely automated and no driver exists anymore, there will be no reason for administrative requirements for car driving, and no driver will be held criminally liable. Concerning civil liability, the introduction of driverless cars is already causing a shift from the paradigms of tort liability (Colonna, 2016) and insurance (Sloan, 1994) to product liability (Villasenor, 2014). Negligence will still be one

2 A partial overview of the legal requirements for cars in the European Union and the United States can be found at http://europa.eu/youreurope/citizens/vehicles/index_en.htm and https://www.usa.gov/cars respectively. of the perspectives under which vehicle liability is viewed, but with a significant shift: from the driver for being inattentive or in conditions not suitable for driving, to the car owner (Duffy and Hopkins, 2013) or the manufacturer (Gurney, 2013) for defects in maintenance or in the manufacturing process.

This is the legal context that can be expected in a future, when damage from a car will be treated just like damage from a microwave oven. But the current transition phase presents even more complex challenges. The technology behind driverless cars is undergoing extensive testing and refinements, and current driverless cars have a dual mode, allowing the human occupant to take manual control of the vehicle. Such a situation calls for the combination of the two approaches, the one based on the drive's responsibility for damages and criminal offences, and the one based on the manufacturer's liability for defective products. Additional questions arise from the interaction between driverless and human-driven cars, which is inherently dangerous as has been shown by several accidents, even with fatal consequences (Singhvi and Russell, 2016).

Significant questions posed in light of the first cause concern the degree of liability of the human occupant. For example, in those cases where the occupant's reaction in taking control of the vehicle would be deemed to have the potential to avoid an accident, he or she might be held liable for negligence. The second cause, on the other hand, calls for an investigation of the degree of liability that can be placed on the driver of the human-driven car and on the product liability of the driverless car. Determining the degree of liability that can be placed on the human driver or on the car manufacturer will be a major challenge in courts, as the increasing adoption of autonomous vehicles will also increase the accident rate.

Finally, it is evident that this shift will also have implications on the insurance system. Today, most countries require cars to be provided with insurance. But as the emphasis shifts from driver liability to product liability, a similar shift in insurance will accommodate the change (Thierer and Hagemann, 2015).

\section{Conclusions}

In this paper, a general technical and functional overview of the recently founded Research Center for Autonomous Road Vehicles (RECAR) was provided. The main focus of the new establishment is education and research in order to provide sufficiently trained engineers for autonomous research. Also, technical environment is required, thus the laboratory structure and basics of a new test track were discussed. Considering research areas, the main areas of autonomous vehicle technology have also been identified, which are the development of autonomous vehicle demonstration platform, the control of driverless vehicles, the communication systems within and among vehicles, the environment sensing of autonomous vehicles, the intelligent transportation systems, the interaction of human and autonomous vehicles, and the testing and validation 
of automated vehicles. Also, the specification of a test track was discussed. Concerning the test track facilities, a concise specification was provided including general requirements and specific framework for road traffic control tests. Finally, the legal framework was presented where administrative requirements and liability issues were discussed.

\section{Acknowledgement}

The work was supported by the ÚNKP-16-4-III New National Excellence Program of the Ministry of Human Capacities.

\section{References}

Bansal, P., Kockelman, K. M. (2017). Forecasting Americans' long-term adoption of connected and autonomous vehicle technologies. Transportation Research Part A: Policy and Practice. 95, pp. 49-63. https://doi.org/10.1016/j.tra.2016.10.013

Colonna, K. (2012). Autonomous Cars and Tort Liability. Case Western Reserve Journal of Law Technology \& Internet. 4(4), pp. 81-131.

Department for Transport. (2015). The pathway to driverless cars: a code of practice for testing, Great Mister House, 33 Horseferry Road, London SW1P4DR, July 2015. URL: https:/www.gov.uk/government/uploads/ system/uploads/attachment_data/file/446316/pathway-driverless-cars.pdf

Duffy, S. H., Hopkins, J. F. (2013). Sit, Stay, Drive: The Future of Autonomous Car Liability. SMU Science \& Technology Law Review. 101, pp. 101-123.

Fagnant, D. J., Kockelman, K. K.(2015). Preparing a nation for autonomous vehicles: opportunities, barriers and policy recommendations. Transportation Research Part A: Policy and Practice. 77, pp. 167-181. https://doi.org/10.1016/j.tra.2015.04.003A

Gáspár, P., Szalay, Zs., Aradi, Sz. (2014). Highly Automated Vehicle Systems. BME MOGI, 2014. URL: http://www.mogi.bme.hu/TAMOP/jarmurendszerek_iranyitasa_angol/index.html

Gurney, J. K. (2013). Sue My Car Not Me: Products Liability and Accidents Involving Autonomous Vehicles. University of Illinois Journal of Law, Technology \& Policy. 2013, pp. 247-277.

Harper, F. V., Kime, P. M. (1934). The Duty to Control the Conduct of Another. Yale Law Journal. 43(6), pp. 886-905.

Hoeger, R., Zeng, Z., Hoess, A. (2011). HAVEit- The future of driving, Final Report, Continental Automotive GmbH and partners. URL: http://haveit-eu.org/LH2Uploads/ItemsContent/24/HAVEit_212154_D61.1_Final_ Report_Published.pdf
Marchant, G. E., Lindor, R. A. (2012). The Coming Collision between Autonomous Vehicles and the Liability System. Santa Clara Law Review. 52(4), pp. 1321-1340.

Milakis, D., Snelder, M., Van Arem, B., Van Wee, B, Correia, G. (2017). Development of automated vehicles in the Netherlands: scenarios for 2030 and 2050. European Journal of Transport and Infrastructure Research, EJTIR. 17(1), pp. 63-85. URL: http://www.tbm.tudelft.nl/fileadmin/Faculteit/TBM/Onderzoek/EJTIR/Back_issues/17.1/2017_01_03.pdf

Pascàud, M. H. (1908). La Circulation des Automobiles et la Responsabilité Penale et Civile de Leurs Conducteurs et Proprietaires. (Traffic of automobiles and the criminal and civil liability of their chauffeurs and owners.) Revue Critique de Législation et de Jurisprudence. 37, pp. 602-625. (in French)

SAE International. (2014). Taxonomy and Definitions for Terms Related to On-Road Motor Vehicle Automated Driving Systems, SAE standard, nr. J3016_201401. URL: http://standards.sae.org/j3016_201401

Schoettle, B., Sivak, M. (2015). A preliminary analysis of real-world crashes involving self-driving vehicles. Transportation Research Institute, University of Michigan, Report No. UMTRI-2015-34.

Singhvi, A., Russell, K. (2016). Inside the Self-Driving Tesla Fatal Accident.

Sloan, F. A., Reilly,B. A., Schenzler, C. M. (1994). Tort liability versus other approaches for deterring careless driving. International Review of Law and Economics. 14(1), pp. 53-71.

https://doi.org/10.1016/0144-8188(94)90036-1

Stapleton, J. (1994). Product Liability. Butterworth-Heinemann.

Szalay, Z. (2016). Structure and Architecture Problems of Autonomous Road Vehicle Testing and Validation". In: 15th Mini Conference on Vehicle System Dynamics, Identification and Anomalies - VSDIA 2016, Budapest, Hungary, 7-9 November 2016.

Thierer, A. D., Hagemann, R. (2015). Removing Roadblocks to Intelligent Vehicles and Driverless Cars. Wake Forest Journal of Law Policy. 5(2), pp. pp. 339-391.

Törő, O., Bécsi, T., Aradi, S. (2016). Design of Lane Keeping Algorithm of Autonomous Vehicle. Periodica Polytechnica Transportation Engineering. 44(1), pp. pp. 60-68. https://doi.org/10.3311/PPtr.8177

Villasenor, J. (2014). Products Liability and Driverless Cars: Issues and Guiding Principles for Legislation. Center for Technology Innovation at Brookings.

Waldrop, M. M. (2015). Autonomous vehicles: No drivers required. Nature. 518(7537), pp. 20-23. https://doi.org/10.1038/518020a 